Trauma Berufskrankh 2007 - 9[Suppl 3]:

S380-S383

DOI 10.1007/s10039-007-1241-4

Online publiziert: 29. Juni 2007

(c) Springer Medizin Verlag 2007
S. Arens

Klinik für Orthopädie und Unfallchirurgie, HELIOS-Klinikum, Siegburg

\section{Postoperative Antibiotika}

\section{Hilfe oder Beruhigung?}

Die Einführung der Antibiotika zur Behandlung bakterieller Infektionen Mitte des letzten Jahrhunderts hat den natürlichen Verlauf dieser weit verbreiteten und gefährlichen Erkrankungen revolutioniert. Dies gilt auch für die Behandlung von Wundinfektionen nach operativen Eingriffen.

In den ersten Dekaden der mit der Einführung des Penizillins etwa 1943 beginnenden „Antibiotikaära“ zeigten sich solch überwältigende klinische Erfolge der Antibiotikabehandlung, dass die Atmosphäre des nahezu ungebremsten Optimismus und blinden Vertrauens in die Erfolgsgarantie dieser Substanzen z. T. bis heute andauert - und zwar sowohl auf Seiten der medizinischen Laien als auch in der Ärzteschaft [16].

Egal ob präoperativ oder postoperativ es wurde in diesen Jahrzehnten kaum gezweifelt: Antibiotika waren Hilfe und Beruhigung.

Erst Ende der 1980er Jahre wurden beunruhigende Probleme der Antibiotikaanwendung bekannt, die dieses ErfolgsCredo ins Wanken brachten und Gefahren aufzeigten:

- Resistenzentwicklungen, die eine „ungezielte und breite" Initialtherapie mit Antibiotika immer häufiger fehlschlagen und teilweise bereits „Reserveantibiotika" unwirksam werden lassen,

- Kostensteigerungen bei der Entwicklung immer neuer, teurerer und toxischerer antibakterieller Substanzen, die nur noch von wenigen reichen Industriestaaten finanzierbar sind,

- zunehmende antibiotikaassoziierte Morbidität, wie Enterokolitis durch Clostridium difficile, Nephro- und Ototoxizität oder Allergien.
Der Kliniker gerät bei seiner Entscheidung für oder gegen die Anwendung postoperativer Antibiotika innerhalb diesen beiden Antipoden rasch in ein Dilemma zwischen Patientenforderungen, juristischer „Be-Drohung“, Pharma-Lobby, unsicherer wissenschaftlicher Datenlage, mangelnden Leitlinien oder auch eigenen „alten“ Gewohnheiten. Bei der postoperativen Antibiotikagabe konkurriert das subjektive Sicherheitsbedürfnis mit der objektiv gesicherten Indikation.

\section{Indikationen für die Antibiotikagabe}

Die klassischen Indikationen für eine Gabe von Antibiotika im zeitlichen Zusammenhang mit einer Operation sind:

- die Prophylaxe und

- die Therapie.

Der Einsatz eines Antibiotikums zur Therapie einer bereits vorhandenen Infektion, also bei Eingriffen der CDC-Wundkategorie VI („dirty“/“infected“), ist unumstrittener fester Bestandteil des multimodalen peri- und postoperativen Behandlungskonzepts einer chirurgischen Infektion.

Das Wesen der Prophylaxe zeichnet sich dadurch aus, dass sie perioperativ bei Abwesenheit einer chirurgischen Infektion zum Einsatz kommt, also bei aseptischen Eingriffen oder solchen mit bakterieller Kontamination - jedoch ohne das Vorliegen einer manifesten Infektion [19].

\section{Perioperative Antibiotikaprophylaxe}

Ihr Ziel ist die Senkung der Rate postoperativer Infektionen, unabhängig von der
Art des operativen Eingriffs. Angestrebt wird die Minimierung der mikrobiellen Belastung - nicht die „Sterilisation“ des Operationsgebiets.

\section{Indikation}

Allgemein formuliert ist sie in folgenden Situationen gegeben:

- Hohes Risiko einer intraoperativen Kontamination mit nachfolgender postoperativer Infektion durch systemischen Keimeintrag (z. B. als „kontaminiert“ klassifizierte Operationen)

- Geringes Risiko einer Infektion, aber erhebliche Konsequenzen in Bezug auf Morbidität oder gar Letalität, wenn es doch zu einer solchen kommt (z. B. Implantate, Fremdmaterial, Endoprothesen, Eingriffe an großen Gelenken)

- Dem Patienten selbst wohnen erhebliche Risikofaktoren inne (z. B. Immunsuppression, potenzieller Focus alio loco, Endokarditis usw.).

\section{Grundlagen}

Die pathophysiologischen Grundlagen der perioperativen Antibiotikaprophylaxe wurden bereits vor mehr als 50 Jahren durch experimentelle Untersuchungen von Miles et al. [20] und Burke [5] erarbeitet. Sie demonstrierten die Bedeutung des perioperativen Zeitpunkts der prophylaktischen Antibiotikagabe und bestätigten die Unzulänglichkeit von erst postoperativ verabreichten Substanzen. Bereits damals wurde erkannt, dass es zur Infektionsvermeidung erforderlich ist, dass schon zum Zeitpunkt der mikrobiellen Gewebekontamination ein ausreichend hoher antibiotischer Wirkspiegel im Gewebe vorhanden 
ist. Systemische Antibiotika, die erst postoperative, d. h. nach dem Kontaminationsereignis, verabreicht wurden, hatten keinen spürbaren Effekt auf die Infektentstehung.

Spätestens seit den Arbeiten von Gristina [12] in den 1980er Jahren sollten die Gründe für das Versagen postoperativer Antibiotika zur Prophylaxe bekannt sein. Mikroorganismen in chirurgischen Wunden werden intraoperativ in einer Fibrinmatrix oder/und einem Biofilm eingebettet, die bereits zum Zeitpunkt des Wundverschlusses entstehen. Antibiotika, die dann nicht bereits „vor Ort" sind, können diese Abwehrmatrix nicht mehr durchdringen und erreichen die Mikroorganismen nicht. Hinzu kommt im Operationsgebiet eine Zone relativer Minderperfusion, die durch lokales Trauma, Ödem und Druck bedingt ist, sodass Antibiotika gar nicht erst in diese relativ ischämische Problemregion transportiert werden.

\section{Anwendung - Leitlinien und Praxis}

Konsequenterweise nehmen in so genannten „Leitlinien“ zur perioperativen Antibiotikaprophylaxe - neben Hinweisen zu Substanz, Dosierung und Applikationsweg - der „perioperative“ Zeitpunkt und die Dauer der Applikation einen wichtigen Stellenwert ein. In den „AWMF-Leitlinien zur Hygiene in Klinik und Praxis“ [2] wird präzisiert:

„Das Prophylaxefenster umfasst den Zeitraum... bis Operationsende“.

Zur Dauer der Prophylaxe heißt es: „Eine über die Einmaldosis hinausgehende Antibiotikaverabreichung hat in keiner wissenschaftlichen Studie eine belegt höhere Effizienz“.

Als strenge Ausnahmen werden eine die Halbwertszeit des Antibiotikums überschreitende Operationszeit von $>3-$ $5 \mathrm{~h}$, die Endokarditisprophylaxe und eine $>12 \mathrm{~h}$ verlängerte Prophylaxe bei offenen Frakturen genannt.

In der klinischen Alltagspraxis scheint das jedoch nicht überall auf diese Weise gehandhabt zu werden $[15,22]$. Nicht nur im eigenen Land [7], sondern auch in anderen europäischen Ländern und den USA $[3,4,9,16,21]$ zeigte sich die Compliance der Operateure mit den nationalen „Leitlinien“ zur Antibiotikaprophylaxe „suboptimal“. In bis zu $41 \%$ wur- de eine „verlängerte“ Antibiotikaprophylaxe durchgeführt - aufgrund individueller Praxis der Operateure! oder zu ihrer Beruhigung?

Fonseca et al. [8] wollten in einer kürzlich publizierten klinischen Studie an $>12.000$ Patienten die "Compliance“ der Chirurgen mit einer „single dose“Antibiotikaprophylaxe in ihrem eigenen Krankenhaus überprüfen und den Operateuren nachweisen, dass diese Einmaldosis (Cephazolin) im Vergleich zu einer „verlängerten“ Antibiotikaprophylaxe nicht zu einem Anstieg der Infektionsraten führt. Unterstützt durch ein Schulungsprogramm gelang es, die „single-dose“-Prophylaxe mit einer 99\%igen Compliance-Rate einzuführen - bei unveränderter Infektionsrate von $2 \%$ in der Gesamtheit aller operativer Fachgebiete einschließlich orthopädischer Chirurgie. Eine zusätzliche Überzeugungshilfe war das Ergebnis, dass durch die einmalige Gabe erhebliche Kosten allein für Antibiotika (ohne Infusionsbestecke und Arbeitskraft) eingesparten wurden.

\section{Antibiotika im Rahmen der operativen Frakturbehandlung - peri- und/oder postoperativ}

Zum Thema der perioperativen Antibiotikaprophylaxe bei geschlossenen Frakturen stehen uns ausreichend Literaturdaten zur Verfügung, insbesondere eine aktuelle Metaanalyse der Cochran Collaboration von Gillespie u. Walenkamp [10]. Eingeschlossen wurden 8307 Fälle von geschlossenen Frakturen des proximalen Femurs und anderen lange Röhrenknochen in 22 Studien, die die Einschlusskriterien erfüllten. Die Metaanalyse kam zu folgenden Aussagen:

- Die Antibiotikaprophylaxe wird bei der offenen Reposition und internen Stabilisierung geschlossener Frakturen empfohlen.

- Eine „single-dose“-Prophylaxe senkt die Rate postoperativer Infektionen signifikant.

- Eine „multiple-dose“-Prophylaxe zeigt den gleichen Effekt, aber keinen weiteren Vorteil.

- Bei ausreichender Datensicherheit sind weiter Studien zu diesem Thema ethisch nicht zu rechtfertigen!
Trauma Berufskrankh $2007 \cdot 9$ [Suppl 3]: S380-S383

DOI 10.1007/s10039-007-1241-4

C) Springer Medizin Verlag 2007

\section{S. Arens}

\section{Postoperative Antibiotika. Hilfe oder Beruhigung?}

\section{Zusammenfassung}

Die Ära, in der Antibiotika in der postoperativen Phase unkritisch zur Hilfe und Beruhigung eingesetzt werden konnten, ist vorbei. Heute kennen wir beunruhigende, antibiotikaassoziierte Gefahren: Resistenzentwicklung, Kostensteigerung bei der Neuentwicklung und antibiotikainduzierte Morbidität. Im Rahmen der Prophylaxe ist die postoperative Gabe von Antibiotika bis auf seltene Ausnahmen nicht indiziert. Es gibt keine sicheren Daten, die den Nutzen einer über die präoperative Einmaldosis herausgehenden Verlängerung der Antibiotikaprophylaxe belegen. In Rahmen der operativen Therapie einer bereits eingetretenen Infektion ist die postoperative Gabe von Antibiotika lediglich ein Bestandteil des prädominant chirurgischen Behandlungskonzepts.

\section{Schlüsselwörter}

Antibiotikum · Antibiotikaprophylaxe · Antibiotikatherapie · Nosokomiale Infektion · Postoperative Infektion

\section{Postoperative antibiotics. A real help, or just reassurance?}

\section{Abstract}

The times when antibiotics could be used indiscriminately to provide help and reassurance in the postoperative period are definitely over. We are now aware of quite alarming threats associated with antibiotics: resistance, cost increases with new developments and antibiotic-induced morbidity. Antibiotics are not indicated postoperatively for prevention of infection except in exceptional cases. No reliable data are available to document any advantage of extending antibiotic prohylaxis beyond the usual single preoperative dose. In the context of surgical treatment of an already manifest infection the postoperative administration of antibiotics is merely one element in the predominantly surgical treatment strategy.

\section{Keywords}

Antibiotic - Preventive antibiotic treatment . Antibiotic treatment $\cdot$ Nosocomial infection . Postoperative infection 
Infobox 1: Kampagne zur Vermeidung

von Resistenzentwicklung

- CDC: campaign to prevent antimicrobial resistance in healthcare settings: http:// www.cdc.gov/drugresistance/healthcare/ default.htm

Auch zur Frage der Antibiotikaprophylaxe bei offenen Extremitätenfrakturen liegt eine aktuelle Metaanalyse der Cochran Collaboration von Gosselin et al. [11] vor. Die strengen Gütekriterien an das Studiendesign erlaubten den Einschluss von lediglich 7 Studien mit insgesamt 913 Fällen. Die Analyse kam zu der Schlussfolgerung, dass Antibiotika zur Prophylaxe die Infektinzidenz bei offenen Frakturen senken. Eine eindeutige Aussage zur Häufigkeit und Dauer der Antibiotikagabe wurde nicht gemacht.

$\mathrm{Zu}$ diesem Punkt wurde aber in der Publikation der „Surgical Infection Society" von Hauser et al. [13] eindeutig Position bezogen:

- Die „single-dose“-Prophylaxe mit einem Zephalosporin der 1. Generation ist effektiv.

- Die kurze Prophylaxe ist äquivalent zur „verlängerten“ postoperativen Prophylaxe bis $48 \mathrm{~h}$.

- Es gibt keine Daten, die belegen, dass die verlängerte, postoperative Antibiotikagabe die Infektionsinzidenz senkt.

Hauser et al. [13] warnten in dieser Arbeit jedoch ausdrücklich, dass es erhebliche Risiken einer „verlängerten“ postoperativen Antibiotikaprophylaxe gibt, insbesondere ein erhöhtes Risiko der Infektion mit nosokomialen, resistenten Mikroorganismen. Unter dem Selektionsdruck einer prolongierten Antibiotikaexposition werden unproblematische Mikroorganismen abgetötet, sodass die initial wenigen, resistenten Keime sich ungehemmt vermehren und erheblichen Krankheitswert, insbesondere hinsichtlich nosokomialer Pneumonien, entwickeln. Velmahos et al. [23] wiesen bereits 2002 eindeutig darauf hin, dass auch ein schweres Trauma keine Entschuldigung für eine prolongierte Antibiotikaprophylaxe ist.

Im Bereich der elektiven Knochenchirurgie einschließlich der Endoprothetik hat sich auch in der aktuellen deutschsprachigen Literatur die Auffassung durchgesetzt, dass eine „verlängerte“, postopera- tive Antibiotikagabe zur Prophylaxe nicht indiziert ist $[15,22]$.

\section{Therapie}

Die postoperative Gabe eines Antibiotikums im Rahmen der Therapie einer chirurgischen Infektion ist wesentlicher Bestandteil eines multimodalen Therapiekonzepts zur Infektbehandlung $[1,14,17]$. Klar zu betonen ist die vorrangige Bedeutung der operativ-chirurgischen Infektsanierung nach dem uralten Prinzip „ubi pus, ibi evacua“! Chirurgisches Débridement und repetitive Nekrektomie von allem avitalen Gewebe haben erste Präferenz [24]. Die kombinierte adjuvante antibiotische Chemotherapie wird durchgeführt nach den Prinzipien:

- gezielt nach Antibiogramm,

- so kurz wie möglich und

- risikobewusst.

Das Risikobewusstsein zielt hierbei auf die eingangs skizzierte Problematik der Resistenzentwicklung mit all ihren Folgen für den Kliniker. Ausdrücklich möchten wir in diesem Zusammenhang die vom CDC initiierte weltweite Kampagne zu dieser Problematik erwähnen und auf die entsprechende Referenz im Internet verweisen ( $\bullet$ Infobox 1).

\section{Fazit für die Praxis}

Jeder Operateur, der Antibiotika zur Prophylaxe und Therapie einer Infektion einsetzt, muss wissen und berücksichtigen, dass diese Substanzen nicht nur eine Hilfe sein können. Ihre Anwendung kann rasch zur Gefahr werden, aufgrund katalysierter Resistenzentwicklungen und der damit verbundenen globalen Gefahren. Die postoperative Gabe eines Antibiotikums lediglich „zur Beruhigung“ von Patient und Operateur ist gefährlich und falsch.

Im Rahmen der Prophylaxe hat eine über die Einmaldosis hinausgehende postoperative Antibiotikagabe in keiner wissenschaftlichen Studie eine belegt höhere Effizienz als die präoperative „single-dose"-Prophylaxe. Die korrekte Durchführung der präoperativen prophylaktischen Antibiotikagabe bezüglich Zeitpunkt und Applikationsweg ist dabei al- lerdings von hoher Bedeutung $[6,16]$. Ausnahmen sind eine die Halbwertszeit des Antibiotikums überschreitende Operationszeit von >3-5 h oder die Endokarditisprophylaxe.

Im Rahmen der Therapie ist die postoperative Antibiotikagabe ein wichtiger und hilfreicher Bestandteil in Ergänzung des prädominant chirurgischen Gesamtbehandlungskonzepts - mehr nicht.

\section{Korrespondenzadresse}

\section{PD Dr. S. Arens}

Klinik für Orthopädie und Unfallchirurgie, HELIOS-Klinikum Siegburg,

Ringstraße 49, 53721 Siegburg

stephan.arens@helios-kliniken.de

Interessenkonflikt. Der korrespondierende Autor gibt an, dass kein Interessenkonflikt besteht.

\section{Literatur}

1. Anders A, Anders S, Gatermann SG (2002) Mikrobiologische Diagnostik und Antibiotikatherapie der Osteitis. Trauma Berufskrankh 4: 314-320

2. AWMF (2004) AWMF-Leitlinien (Nr. 029/022): Perioperative Antibiotikaprophylaxe. Hygiene in Klinik und Praxis, 3. Aufl. mph, Wiesbaden, S 139ff, http://www.uni-duesseldorf.de/AWMF/II/029-022. htm

3. Barie PS (2000) Modern surgical antibiotic prophylaxis and therapy - less is more. Surg Infect 1:2329

4. Bull AL, Russo PL, Friedman ND et al. (2006) Compliance with surgical antibiotic prophylaxis - reporting from a statewide surveillance programme in Victoria, Australia. J Hosp Infect 63: 140-147

5. Burke JF (1961) The effective period of preventive antibiotic action in experimental incisions and dermal lesions. Surgery 50: 161-168

6. Dellinger EP (2007) Prophylactic antibiotics: administration and timing before operation are more important than administration after operation. Clin Infect Dis 44: 928-930

7. Dettenkofer M, Forster DH, Ebner W et al. (2002) The practice of perioperative antibiotic prophylaxis in eight German hospitals. Infection 30: 164167

8. Fonseca SN, Kunzle SR, Junqueria MJ et al. (2006) Implementing 1-dose antibiotic prophylaxis for prevention of surgical site infection. Arch Surg 141: 1109-1113

9. Gagliotti C, Ravaglia F, Resi D et al. (2004) Quality of local guidelines for surgical antimicrobial prophylaxis. J Hosp Infect 56: 67-70

10. Gillespie WJ, Walenkamp G (2007) Antibiotic prophylaxis for surgery for proximal femoral and other closed long bone fractures. The Cochrane Library. Cochrane Collab 1

11. Gosselin RA, Roberts I, Gillespie WJ (2007) Antibiotics for preventing infection in open limb fractures. The Cochrane Library. Cochrane Collab 1

12. Gristina AG (1987) Biomaterial-centred infection: microbial adhesion versus tissue integration. Science 237: 1588-1595 
13. Hauser CJ, Adams CA Jr, Eachempati SR, Council of the Surgical Infection Society (2006) Surgical Infection Society guideline: prophylactic antibiotic use in open fractures: an evidence-based guideline. Surg Infect 7: 379-405

14. Heppert V, Wagner C, Glatzel U et al. (2002) Prinzipien der operativchirurgischen Therapie der Osteitis. Trauma Berufskrankh 4: 321-328

15. Hunfeld KP, Wichelhaus TA, Schafer V et al. (2003) Perioperative Antibiotikaprophylaxe bei aseptischen Eingriffen in der Orthopädie. Orthopäde 32: 1070-1077

16. Kasteren ME van, Mannien J, Kullberg BJ et al. (2005) Quality improvement of surgical prophylaxis in Dutch hospitals: evaluation of a multi-site intervention by time series analysis. J Antimicrob Chemother 56: 1094-1102

17. Kutscha-Lissberg F, Hebler U, Kälicke T et al. (2002) Inzidenz, Diagnose, Manifestations- und Verlaufsformen der Osteitis. Trauma Berufkrankh 4: 297305

18. Lazzarini L, Lipsky BA, Mader JT (2005) Antibiotic treatment of osteomyelitis: what have we learned from 30 years of clinical trials? Int J Infect Dis 9: $127-138$

19. Mangram AJ, Horan TC, Pearson ML et al. (1999) Guideline for prevention of surgical site infection, 1999. Centers for Disease Control and Prevention (CDC) Hospital Infection Control Practices Advisory Committee. Am J Infect Control 27: 97-132

20. Miles A, Miles E, Burke J (1957) The value and duration of defence reactions of the skin to the primary lodgement of bacteria. Br J Exp Pathol 38: 79-96

21. Quenon JL, Eveillard M, Vivien A et al. (2004) Evaluation of current practices in surgical antimicrobial prophylaxis in primary total hip prosthesis - a multicentre survey in private and public French hospitals. J Hosp Infect 56: 202-207

22. Szell M, Hofmann S, Pietsch M et al. (2006) Perioperative antibiotische Prophylaxe. Einsatz in der Orthopädie. Orthopäde 35: 805-812

23. Velmahos GC, Toutouzas KG, Sarkisyan G et al. (2002) Severe trauma is not an excuse for prolonged antibiotic prophylaxis. Arch Surg 137: 537542

24. Wick M, Kutscha-Lissberg F, Kollig E et al. (2002) Debridierungstechniken bei der operativen Sanierung der Osteitis. Trauma Berufskrankh 4: 358-362 